\title{
Cytochrome P450 family 4 subfamily $F$ member 2 (CYP4F2) rs1558139, rs2108622 polymorphisms and susceptibility to several cardiovascular and cerebrovascular diseases
}

\author{
Tao Zhang ${ }^{1^{*}}$ (D) Kuiying $\mathrm{Yu}^{1}$ and Xuhua $\mathrm{Li}^{2}$
}

\begin{abstract}
Background: Inconsistent conclusions have been reported for the genetic relationship between CYP4F2 (Cytochrome P450 Family 4 Subfamily F Member 2) polymorphisms and the susceptibility to cardiovascular and cerebrovascular diseases.

Methods: We performed a meta-analysis to assess the potential role of rs1558139 C/T and rs2108622 G/A polymorphisms of CYP4F2 in the risks of cardiovascular and cerebrovascular diseases. The retrieval of four databases, including PubMed, Web of Science (WOS), China National Knowledge Infrastructure (CNKI) and WANFANG DATA, was conducted. Mantel-Haenszel statistics for association test, Cochran's Q statistic, sensitivity analysis for heterogeneity assessment, and Begg's/Egger's tests for publication bias evaluation were performed under allele, homozygote, heterozygote, dominant, and recessive models, respectively.

Results: A total of 597 articles were initially obtained by database searching, and twenty eligible articles were finally included. For rs1558139, a decreased risk of cardiovascular and cerebrovascular diseases was observed in the overall meta-analysis and in "hypertension", "population-based" and "male" subgroups under models of T vs. C, CT vs. CC, and $C T+\Pi$ vs. CC [all $P$ values in association tests $<0.05$, odds ratio $(O R)<1$ ]. For rs 2108622 , a decreased coronary artery disease (CAD) risk was observed in the subgroup meta-analysis based on disease type under all genetic models (all $P$ values in association tests $<0.05, \mathrm{OR}<1$ ). Begg's/Egger's tests excluded the potential publication bias, while sensitivity analysis data supported the stability of the above results.
\end{abstract}

Conclusion: C/T genotype of CYP4AF2 rs1558139 may be linked to the decreased risk of hypertension in the male patients of Asian populations, while CYP4F2 rs2108622 is likely associated with reduced susceptibility to CAD.

Keywords: CYP4F2, Single nucleotide polymorphisms, Coronary artery disease, Hypertension, Ischemic stroke

\section{Background}

Single nucleotide change-induced nucleic acid sequence polymorphisms, namely, single nucleotide polymorphisms (SNPs), occur more frequently in the human genome, with an average of approximately one polymorphism per 1000 bases, and functional SNPs may help to guide population genetics research, as well as the mechanistic, epidemiologic

\footnotetext{
* Correspondence: zbsjnk@163.com

${ }^{1}$ First Department of Neurology, The First Hospital of Zibo, No.4 Emei Mountain Road, Boshan District, Zibo City, Shandong 255200, People's Republic of China

Full list of author information is available at the end of the article
}

or diagnostic study of some clinical genetic diseases $[1,2]$. Emerging SNPs of different genes have been implicated in the complicated etiology or pathogenesis of several cardiovascular and cerebrovascular diseases [3-5]. For example, GWAS (genome-wide association studies) data show that some SNPs, such as rs1004467 of the CYP17A1 (Cytochrome P450 Family 17 Subfamily A Member 1) gene or rs2681492 of the ATP2B1 (ATPase Plasma Membrane $\mathrm{Ca}^{2+}$ Transporting 1) gene, are associated with susceptibility to blood pressure or essential hypertension $[6,7]$. The aim of the present study was to investigate the potential role of the CYP4F2 (Cytochrome P450 Family 4 Subfamily 
F Member 2) polymorphism in the risk of cardiovascular and cerebrovascular diseases, such as myocardial infarction (MI), coronary artery disease (CAD), ischemic stroke (IS), cerebral infarction (CI) and hypertension.

Human CYP4F2 gene is located on chromosome 19 and contains 12 introns and 13 exons [8]. There are several common SNPs, such as rs3093100, rs3093105, rs3093166, rs1558139 and rs2108622 [8, 9]. The SNPs of rs3093100, rs3093105, rs3093166, and rs1558139 are located in the intron region of the CYP4F2 gene, while rs2108622 (namely, V433 M, G1347A, and G20597A) is located in the exon region of this gene $[8,9]$. CYP4F2 protein belongs to the human cytochrome P450 superfamily and functions as an essential enzyme for the production of 20-HETE (20hydroxy eicosane arachidonic acid) [10, 11]. Increased 20-HETE levels are associated with vascular oxidative stress, endothelial dysfunction and high peripheral vascular resistance $[10,12]$. As a bioactive eicosanoid and therapeutic intervention target, 20-HETE is involved in several vascular events, such as the modulation of blood pressure, renal function, cerebral blood flow and pulmonary circulation $[10,12]$.

Considering the conflicting conclusions, we quantitatively measured the genetic correlation between CYP4F2 SNPs and the risk of cardiovascular and cerebrovascular diseases via meta-analysis based on the publicly published data. To our knowledge, one meta-analysis of hypertension [13] and another meta-analysis of ischemic stroke [14] were previously reported for CYP4F2 rs2108622. However, no metaanalysis for the other SNPs of the CYP4F2 gene have been reported. Additionally, there is no meta-analysis regarding the role of CYP4F2 rs2108622 in the risk other cardiovascular and cerebrovascular diseases.

\section{Methods}

\section{Database searching}

In accordance with the methodology of "Preferred Reporting Items for Systematic Reviews and Meta-Analyses (PRISMA)" [15], we performed the retrieval of four electronic databases, including PubMed, Web of Science (WOS), China National Knowledge Infrastructure (CNKI) and WANFANG DATA, up to Jan 2018. Considering the limitation of publication space, the searching terms for each database are listed in supplemental online Additional file 1: Table S1.

\section{Selection criteria}

After screening, eligible case-control studies provided the sufficient genotype frequency data of CYP4F2 gene in each case/control group. The following exclusion criteria were considered: a) animal or cell experiments; b) other genes/diseases or non-SNP gene variants; c) meeting abstract; d) meta-analysis; e) review or letter; f) undetected variants; g) the absence of genotype frequency data; h) duplicated articles; and i) not in HWE (Hardy-WeinbergEquilibrium).

\section{Basic information}

We extracted the basic information from the eligible articles, namely, first author, year, ethnicity, genotyping assay, SNPs, genotype frequency, disease type, gender, mean age, study number, sample size of the case/control, $\mathrm{X}^{2}$ and $P$ values of HWE, and source of control. The paper quality was assessed by the Newcastle-Ottawa Scale (NOS) system [16], which indicates poor quality when the NOS score is less than five.

\section{Statistical analysis}

Based on Mantel-Haenszel statistics, the value of OR (odds ratio), 95\% CIs (confidence intervals) and $P$ value of association test was generated under the allele, homozygote, heterozygote, dominant, and recessive models. A fixed-effect model was used when the $P$ value of heterogeneity from Cochran's Q statistic was larger than 0.05 or $\mathrm{I}^{2}$ less than $50.0 \%$. Subgroup meta-analyses by ethnicity (Caucasian/Asian), disease type (hypertension/CAD/IS/ $\mathrm{CI} / \mathrm{MI}$ ), source of control (population/hospital -based), and gender (male/female), were also conducted through Mantel-Haenszel statistics. For publication bias assessment, Begg's/Egger's tests were performed. Stata/SE software version 12.0 (StataCorp LP,College Station, TX 77845 USA) was used for the above statistical analyses.

\section{Results}

\section{Eligible case-control studies}

Eligible case-control studies were obtained following the flow diagram of Fig. 1. We first obtained 597 articles from database searching, namely, PubMed with 128 articles, WOS with 369 articles, CNKI with 36 articles, and WANFANG DATA with 65 articles. Next, we removed 105 articles with duplicated data and 432 articles did not met the inclusion criteria. A total of 60 full-text articles were then assessed for eligibility. We further removed 40 articles for undetected variants or the absence of genotype frequency data. Finally, 20 articles [8, 9, 17-34] were included in the present meta-analysis. The basic information and genotype frequencies of eligible casecontrol studies for the present meta-analysis are provided in Additional file 1: Tables S2-S5. Two polymorphisms within the CYP4F2 gene (rs1558139 C/T and rs2108622 G/A) were analyzed. The NOS system data suggested that no included studies showed poor quality, as all NOS scores were larger than five (Additional file 1: Table S3). Moreover, all the genotype distributions of controls adhered to Hardy-Weinberg Equilibrium (Additional file 1: Table S4-S5). 


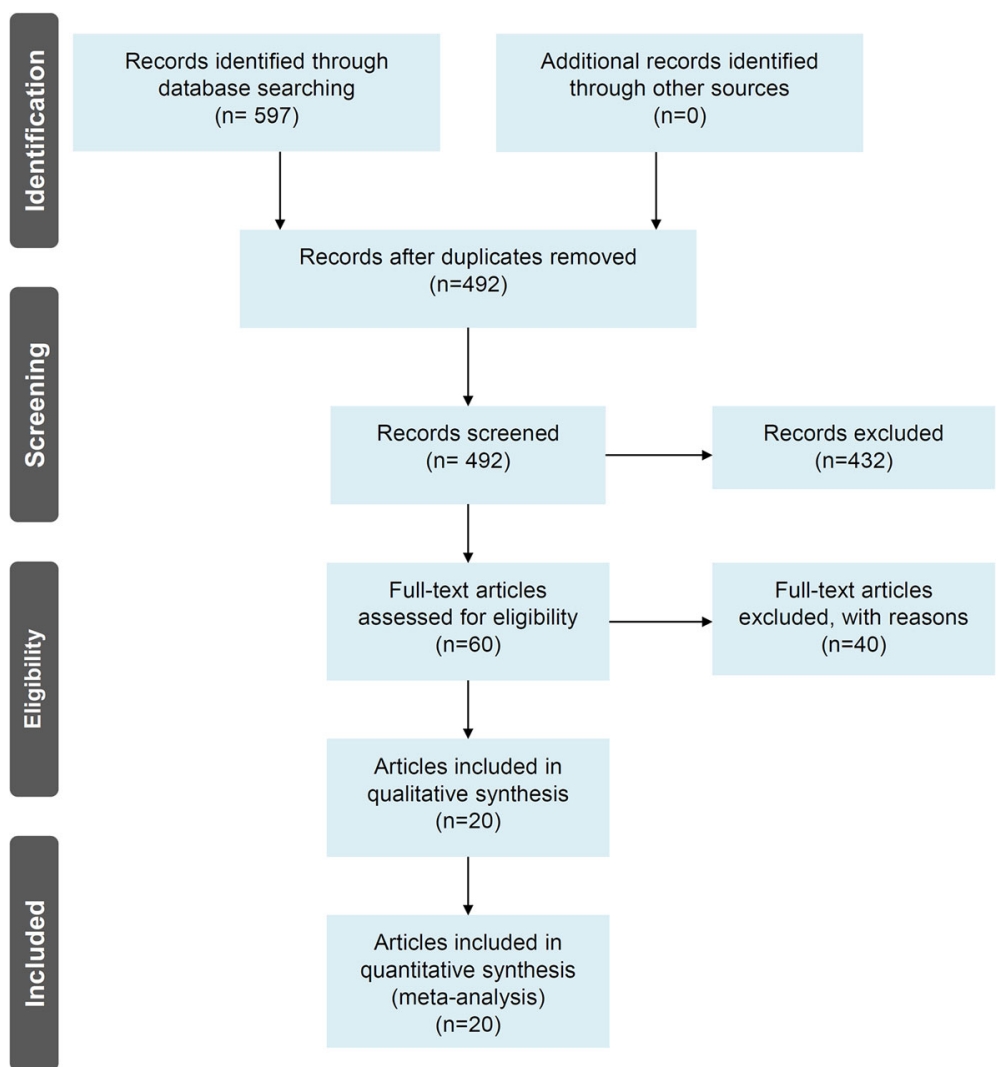

Fig. 1 Flow diagram of the eligible case-control studies identified

\section{The rs $1558139 \mathrm{C} / \mathrm{T}$ polymorphism}

We first conducted a meta-analysis to analyze the association between CYP4AF2 rs1558139 C/T and risk of cardiovascular and cerebrovascular diseases. In Table 1 , ten case-control studies with 3462 cases and 3547 controls in an Asian population were enrolled $[8,18,19,21,22,25,27,33,34]$. Overall meta-analyses and subgroup analyses were conducted under allele ( $G$ vs. A), homozygote (GG vs. AA), heterozygote (AG vs. $A A)$, dominant ( $A G+G G$ vs. $A A)$, and recessive (GG vs. $\mathrm{AA}+\mathrm{AG})$ genetic models. There was no high degree of heterogeneity in any of the meta-analyses (Table $1, \mathrm{I}^{2}$ value $=0.0 \%, P$ value of heterogeneity $>0.05$ ), and fixedeffect model was applied. In pooled data from overall meta-analysis (Table 1), a decreased risk of cardiovascular and cerebrovascular diseases was observed between case and control groups $(\mathrm{T}$ vs. $\mathrm{C}, P$ value in association tests $=$ 0.021, $\mathrm{OR}=0.92$; CT vs. $\mathrm{CC}, P=0.024, \mathrm{OR}=0.89$; $\mathrm{CT}+$ TT vs. $C C, P=0.012$, $\mathrm{OR}=0.88$ ). Moreover, similar results were observed under allele, heterozygote and dominant genetic models in "hypertension" and "population-based" subgroups (Table 1 , all $P$ value in association tests $<0.05$, $\mathrm{OR}<1$ ). We also performed a subgroup analysis based on gender. As shown in Additional file 1: Table S6, a reduced hypertension risk was observed in the "male" subgroup under allele $(P$ value in association tests $<0.001, \mathrm{OR}=$
$0.79)$, homozygote $(P=0.009, \mathrm{OR}=0.68)$, heterozygote $(P<0.001, \mathrm{OR}=0.67)$ and dominant $(P<0.001, \mathrm{OR}=$ $0.67)$ genetic models. Figure 2 shows the forest plot of the subgroup analysis by disease type under the heterozygote model, and Additional file 2: Figure S1 and Additional file 3: Figure S2 presents the data under allele and dominant models. Additional file 4: Figure S3 shows the forest plot of subgroup analysis by gender under the allele model. Thus, these results demonstrated that the $\mathrm{C} / \mathrm{T}$ genotype of CYP4AF2 rs1558139 might be associated with a reduced risk of hypertension in the male patients of Asian populations.

\section{The rs2108622 G/a polymorphism}

Meta-analysis of the association between the CYP4AF2 rs2108622 G/A polymorphism and the risk of cardiovascular and cerebrovascular diseases was subsequently performed. A total of 17 case-control studies were enrolled $[8,9,17-20,23,24,26-32,34]$. As shown in Table 2, a random-effects model was used in all meta-analysis (all $P$ value of heterogeneity $<0.05$ ). A decreased CAD risk was observed in the subgroup meta-analysis by disease type under all genetic models (Table 2, A vs. G, $P$ value in association tests $=0.001, \mathrm{OR}=0.78$; AA vs. GG, $P=0.001$, $\mathrm{OR}=0.55$; GA vs. GG, $P=0.002$, $\mathrm{OR}=0.80$; $\mathrm{GA}+\mathrm{AA}$ vs. 
Table 1 Meta-analysis of CYP4AF2 rs1558139 C/T polymorphism

\begin{tabular}{|c|c|c|c|c|c|c|c|c|c|}
\hline \multirow[t]{2}{*}{ Genetic models } & \multirow[t]{2}{*}{ Subgroup } & \multicolumn{3}{|c|}{ Test of association } & \multirow[t]{2}{*}{$\mathrm{N}$} & \multicolumn{2}{|c|}{ Sample size } & \multicolumn{2}{|c|}{ Heterogeneity } \\
\hline & & OR (95\% Cls) & $z$ & $P$ value & & case & $\overline{\text { control }}$ & $\overline{1^{2}}$ & $P$ value \\
\hline \multirow[t]{4}{*}{ allele (T vs. C) } & overall/Asian & $0.92(0.86,0.99)$ & 2.31 & 0.021 & 10 & 3462 & 3547 & $0.0 \%$ & 0.931 \\
\hline & hypertension & $0.89(0.81,0.98)$ & 2.34 & 0.019 & 4 & 1852 & 1787 & $0.0 \%$ & 0.768 \\
\hline & CAD & $1.00(0.87,1.14)$ & 0.05 & 0.963 & 3 & 883 & 914 & $0.0 \%$ & 0.685 \\
\hline & population-based & $0.91(0.85,0.98)$ & 2.49 & 0.013 & 8 & 2927 & 3033 & $0.0 \%$ & 0.967 \\
\hline \multirow[t]{4}{*}{ homozygote (TT vs. CC) } & overall & $0.87(0.76,1.00)$ & 1.89 & 0.058 & 10 & 3462 & 3547 & $0.0 \%$ & 0.955 \\
\hline & hypertension & $0.85(0.70,1.04)$ & 1.59 & 0.111 & 4 & 1852 & 1787 & $0.0 \%$ & 0.900 \\
\hline & CAD & $0.99(0.76,1.30)$ & 0.05 & 0.963 & 3 & 883 & 914 & $0.0 \%$ & 0.609 \\
\hline & population-based & $0.85(0.70,1.04)$ & 1.99 & 0.047 & 8 & 2927 & 3033 & $0.0 \%$ & 0.991 \\
\hline \multirow[t]{4}{*}{ heterozygote (CT vs. CC) } & overall/Asian & $0.89(0.80,0.98)$ & 2.26 & 0.024 & 10 & 3462 & 3547 & $0.0 \%$ & 0.738 \\
\hline & hypertension & $0.81(0.70,0.93)$ & 2.95 & 0.003 & 4 & 1852 & 1787 & $0.0 \%$ & 0.565 \\
\hline & CAD & $0.98(0.79,1.21)$ & 0.18 & 0.857 & 3 & 883 & 914 & $0.0 \%$ & 0.977 \\
\hline & population-based & $0.87(0.78,0.97)$ & 2.46 & 0.014 & 8 & 2927 & 3033 & $0.0 \%$ & 0.662 \\
\hline \multirow[t]{4}{*}{ dominant (CT + TT vs. CC) } & overall & $0.88(0.80,0.97)$ & 2.53 & 0.012 & 10 & 3462 & 3547 & $0.0 \%$ & 0.866 \\
\hline & hypertension & $0.82(0.71,0.93)$ & 2.95 & 0.003 & 4 & 1852 & 1787 & $0.0 \%$ & 0.650 \\
\hline & CAD & $0.98(0.80,1.20)$ & 0.16 & 0.871 & 3 & 883 & 914 & $0.0 \%$ & 0.952 \\
\hline & population-based & $0.86(0.78,0.96)$ & 2.71 & 0.007 & 8 & 2927 & 3033 & $0.0 \%$ & 0.821 \\
\hline \multirow[t]{4}{*}{ recessive (TT vs. $C C+C T)$} & overall/Asian & $0.93(0.82,1.06)$ & 1.03 & 0.303 & 10 & 3462 & 3547 & $0.0 \%$ & 0.889 \\
\hline & hypertension & $0.95(0.80,1.14)$ & 0.52 & 0.601 & 4 & 1852 & 1787 & $0.0 \%$ & 0.898 \\
\hline & CAD & $1.01(0.80,1.28)$ & 0.11 & 0.914 & 3 & 883 & 914 & $0.0 \%$ & 0.483 \\
\hline & population-based & $0.92(0.80,1.06)$ & 1.14 & 0.256 & 8 & 2927 & 3033 & $0.0 \%$ & 0.977 \\
\hline
\end{tabular}

$O R$ odds ratio, $C l s$ confidence intervals, $C A D$ coronary artery disease, $N$ number of case-control studies

GG, $P=0.005, \mathrm{OR}=0.77 ;$ AA vs. GG $+\mathrm{GA}, P=0.002$, $\mathrm{OR}=0.60$ ). However, no significant associations were observed for other factors (Table 2 and Additional file 1: Table S7). Figure 3 shows the forest plot of the subgroup analysis by disease type under the allele model, and Additional file 5: Figure S4, Additional file 6: Figure S5, Additional file 7: Figure S6 and Additional file 8: Figure S7 presents the data of homozygote, heterozygote, dominant and recessive models. Additional file 9: Figure S8 shows the forest plot of subgroup analysis by gender under the allele model. These data indicated that the CYP4AF2 rs 2108622 G/A polymorphism was associated with reduced susceptibility to CAD.

\section{Publication bias/sensitivity analysis}

Table 3 shows the results of Begg's/Egger's tests under all genetic models $(P$ value in Begg's test $>0.05 ; P$ value in Egger's test $>0.05$ ), suggesting the absence of large publication bias. Fig. 4a presents the funnel plot of Egger's test under the allele model of the CYP4AF2 rs2108622 G/A polymorphism. Furthermore, we observed the stability of the above data based on the sensitivity analysis (Fig. 4b for allele models of rs2108622, other models not shown).

\section{Discussion}

CYP4AF2 rs2108622 SNP was reportedly associated with an increased risk of hypertension cases in India [26] or Northern Han Chinese patients [23]. However, negative data were observed in another study in China [32]. In 2015, Luo et al. performed a meta-analysis of four studies $[8,19,23,32]$ and found that the CYP4AF2 gene rs2108622 polymorphism was not associated with hypertension risk [13]. Here, eight case-control studies $[8,19,20,23,26,27,29,32]$ were included in our metaanalysis, and negative correlation between CYP4AF2 rs2108622 and hypertension risk was observed. Nevertheless, we first investigated the role of CYP4AF2 rs1558139 in the risk of hypertension. The findings suggested that the C/T genotype of CYP4AF2 rs1558139 was correlated with a reduced risk of hypertension in the male patients of Asian populations. As a metabolite of arachidonic acid (AA), partly by CYP4F2 protein, 20-HETE induces the transcription of ACE (angiotensin-converting enzyme) gene through stimulating the NF-KB pathway and contributes to the pathophysiology of hypertension [10]. CYP4AF2 rs1558139, located in an intron region, likely affects the enzyme activity of CYP4F2 protein during 20HETE metabolism through altering the gene transcription 


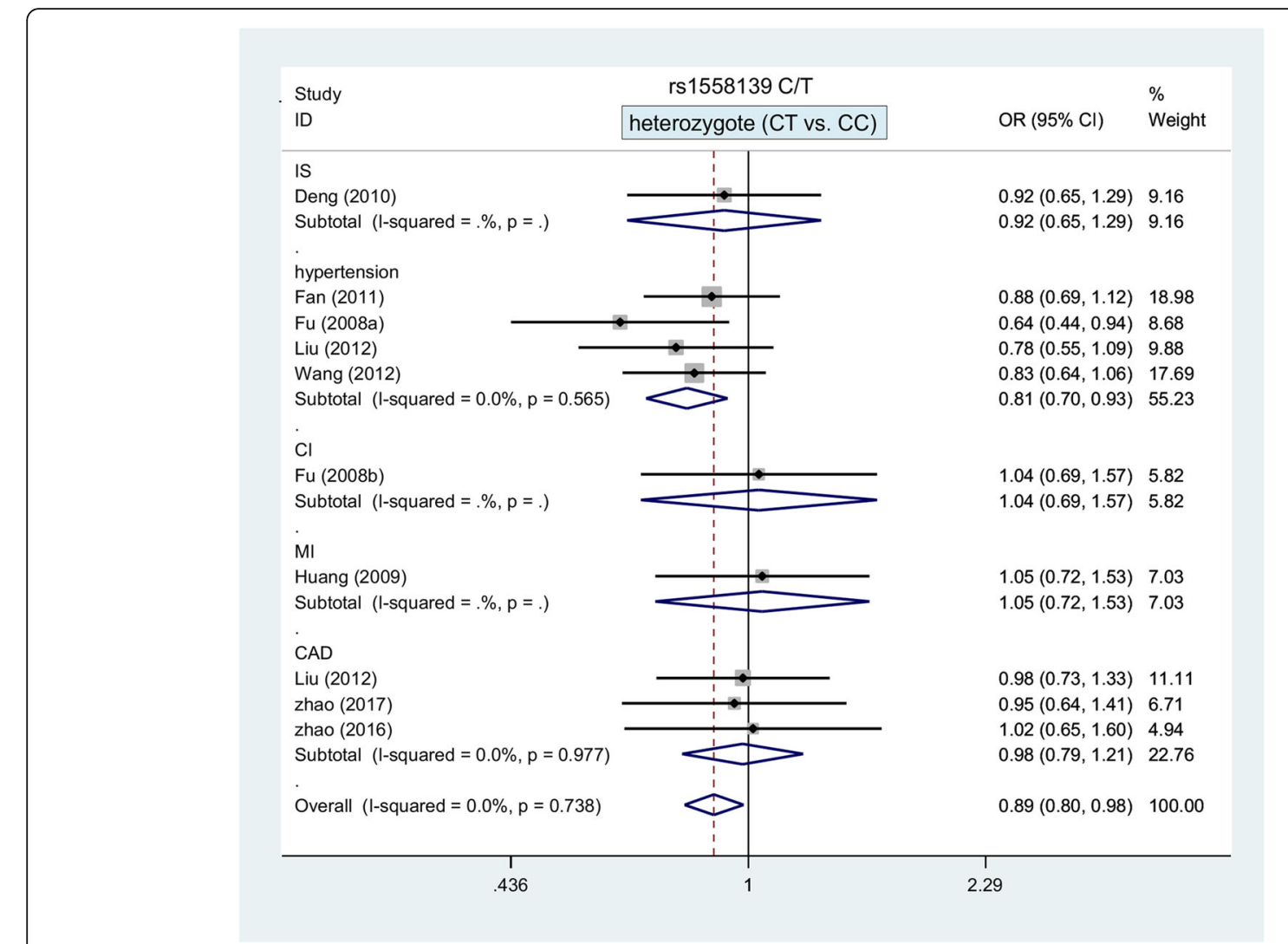

Fig. 2 Subgroup analysis by disease type of the association between the CYP4AF2 rs1558139 polymorphism and the risk of cardiovascular and cerebrovascular diseases under the heterozygote model

level, RNA secondary structure or RNA splicing of the CYP4AF2 gene, which is worth further investigation of the molecular mechanism. In addition, CYP4F2 rs2108622 was tightly associated with systolic blood pressure as well as the excretion of 20-HETE [29]. Additional case-control studies are needed to further confirm the role of CYP4F2 rs2108622 in hypertension risk.

Coronary heart disease results from the interplay between polygenic predisposition and complex environmental factors [35]. The CYP4AF2 gene rs 2108622 polymorphism was associated with the risk of coronary artery disease in a Han Chinese population [9]. However, a negative correlation between the CYP4AF2 rs2108622 and coronary heart disease risk was reported in Mongolian cases in China [34]. In the present study, we first examined whether the CYP4AF2 rs2108622 polymorphism is likely associated with the reduced susceptibility to coronary artery disease. Here, the meta-analysis provided evidence of the involvement of CYP4AF2 rs2108622 in susceptibility to coronary heart disease. Given the reported correlation between CYP4AF2 rs2108622 and 20-HETE [36], functional CYP $4 A F 2$ rs2108622 may mediate the nonsynonymous mutation of a valine $(\mathrm{V})$ located at residue 433 to a methionine
(M), namely, V433 M, which then leads to the alteration of CYP4F2 protein function and 20-HETE synthesis, thereby influencing the occurrence of coronary heart disease.

The early evaluation of genetic risk factors is essential for the effective diagnosis and treatment of clinical ischemic stroke [3, 37]. We observed different conclusions for the association between CYP4AF2 SNPs and ischemic stroke risk. For example, no detectable difference was reported by Liao, et al. for the genotype distribution of CYP4AF2 rs2108622 between the control and cases of ischemic stroke [24]. However, Munshi, et al. reported CYP4AF2 rs2108622 SNP as an important risk factor for ischemic stroke in an Indian population [26]. In 2015, Meng, et al. performed a meta-analysis of ischemic stroke and rs2108622 under the GA + AA vs. GG model [14], enrolling six studies $[18,20,21,26,30,38]$, and reported the data of $\mathrm{OR}>1, P<0.0001$ [14]. In the present study, we removed two studies that did not contain the genotype data $[20,38]$ and one study on cerebral infarction [21], added one new study published in 2016 [24], and finally four studies [18, 24, 26, 30] were enrolled. Additionally, the GA + AA vs. GG model, and other genetic models, 
Table 2 Meta-analysis of CYP4AF2 rs2108622 G/A polymorphism

\begin{tabular}{|c|c|c|c|c|c|c|c|c|c|}
\hline \multirow[t]{2}{*}{ Genetic models } & \multirow[t]{2}{*}{ Subgroup } & \multicolumn{3}{|c|}{ Test of association } & \multirow[t]{2}{*}{$N$} & \multicolumn{2}{|c|}{ Sample size } & \multicolumn{2}{|c|}{ Heterogeneity } \\
\hline & & OR (95\% Cls) & $z$ & $P$ value & & case & $\overline{\text { control }}$ & $\overline{1^{2}}$ & $P$ value \\
\hline \multirow[t]{6}{*}{ allele (A vs. G) } & overall & $0.95(0.87,1.04)$ & 1.05 & 0.295 & 17 & 10,213 & 8033 & $69.9 \%$ & $<0.001$ \\
\hline & Asian & $0.95(0.87,1.04)$ & 1.16 & 0.246 & 15 & 6352 & 5867 & $73.0 \%$ & $<0.001$ \\
\hline & CAD & $0.78(0.68,0.90)$ & 3.40 & 0.001 & 5 & 1934 & 1609 & $38.9 \%$ & 0.162 \\
\hline & IS & $0.93(0.74,1.18)$ & 0.58 & 0.564 & 4 & 1515 & 1545 & $78.0 \%$ & 0.003 \\
\hline & hypertension & $1.07(0.99,1.15)$ & 1.69 & 0.090 & 8 & 6764 & 4879 & $21.8 \%$ & 0.256 \\
\hline & population-based & $0.92(0.81,1.05)$ & 1.26 & 0.209 & 12 & 5203 & 4584 & $73.4 \%$ & $<0.001$ \\
\hline \multirow[t]{6}{*}{ homozygote (AA vs. GG) } & overall & $0.94(0.76,1.16)$ & 0.58 & 0.561 & 17 & 10,213 & 8033 & $64.5 \%$ & $<0.001$ \\
\hline & Asian & $0.90(0.71,1.16)$ & 0.81 & 0.418 & 15 & 6352 & 5867 & $67.9 \%$ & $<0.001$ \\
\hline & $C A D$ & $0.55(0.39,0.77)$ & 3.44 & 0.001 & 5 & 1934 & 1609 & $24.9 \%$ & 0.255 \\
\hline & IS & $0.90(0.56,1.44)$ & 0.45 & 0.651 & 4 & 1515 & 1545 & $73.9 \%$ & 0.009 \\
\hline & hypertension & $0.90(1.02,1.37)$ & 2.29 & 0.022 & 8 & 6764 & 4879 & $0.0 \%$ & 0.525 \\
\hline & population-based & $0.91(0.70,1.19)$ & 0.70 & 0.487 & 12 & 5203 & 4584 & $64.3 \%$ & 0.001 \\
\hline \multirow[t]{6}{*}{ heterozygote (GA vs. GG) } & overall & $0.95(0.89,1.01)$ & 1.69 & 0.092 & 17 & 10,213 & 8033 & $43.9 \%$ & 0.027 \\
\hline & Asian & $0.92(0.85,1.00)$ & 2.06 & 0.039 & 15 & 6352 & 5867 & $48.3 \%$ & 0.019 \\
\hline & $C A D$ & $0.80(0.69,0.92)$ & 3.14 & 0.002 & 5 & 1934 & 1609 & $37.2 \%$ & 0.173 \\
\hline & IS & $0.94(0.80,1.09)$ & 0.84 & 0.400 & 4 & 1515 & 1545 & $47.8 \%$ & 0.125 \\
\hline & hypertension & $1.00(0.93,1.09)$ & 0.09 & 0.927 & 8 & 6764 & 4879 & $19.2 \%$ & 0.278 \\
\hline & population-based & $0.91(0.83,0.99)$ & 2.15 & 0.032 & 12 & 5203 & 4584 & $57.8 \%$ & 0.006 \\
\hline \multirow[t]{6}{*}{ dominant (GA + AA vs. GG) } & overall & $0.93(0.83,1.03)$ & 1.46 & 0.145 & 17 & 10,213 & 8033 & $59.3 \%$ & 0.001 \\
\hline & Asian & $0.91(0.80,1.03)$ & 1.49 & 0.135 & 15 & 6352 & 5867 & $62.9 \%$ & 0.001 \\
\hline & $C A D$ & $0.77(0.64,0.92)$ & 2.83 & 0.005 & 5 & 1934 & 1609 & $43.3 \%$ & 0.133 \\
\hline & IS & $0.93(0.71,1.21)$ & 0.57 & 0.572 & 4 & 1515 & 1545 & $69.4 \%$ & 0.020 \\
\hline & hypertension & $1.03(0.95,1.12)$ & 0.77 & 0.442 & 8 & 6764 & 4879 & $6.0 \%$ & 0.384 \\
\hline & population-based & $0.89(0.77,1.03)$ & 1.52 & 0.130 & 12 & 5203 & 4584 & $68.5 \%$ & $<0.001$ \\
\hline \multirow[t]{6}{*}{ recessive (AA vs. GG + GA) } & overall & $0.98(0.82,1.18)$ & 0.22 & 0.827 & 17 & 10,213 & 8033 & $58.7 \%$ & 0.001 \\
\hline & Asian & $0.95(0.77,1.17)$ & 0.49 & 0.625 & 15 & 6352 & 5867 & $62.5 \%$ & 0.001 \\
\hline & $C A D$ & $0.60(0.44,0.83)$ & 3.06 & 0.002 & 5 & 1934 & 1609 & $19.2 \%$ & 0.293 \\
\hline & IS & $0.93(0.65,1.33)$ & 0.40 & 0.692 & 4 & 1515 & 1545 & $58.8 \%$ & 0.064 \\
\hline & hypertension & $1.22(1.05,1.43)$ & 2.52 & 0.012 & 8 & 6764 & 4879 & $12.5 \%$ & 0.333 \\
\hline & population-based & $0.96(0.77,1.19)$ & 0.39 & 0.694 & 12 & 5203 & 4584 & $50.5 \%$ & 0.023 \\
\hline
\end{tabular}

$O R$ odds ratio, $C l$ s confidence intervals, $C A D$ coronary artery disease, $I S$ ischemic stroke, $N$ number of case-control studies

including A vs. G; AA vs. GG; GA vs. GG; AA vs. GG + GA, were utilized. We obtained a negative result for the genetic relationship between ischemic stroke risk and the CYP4AF2 gene rs2108622 polymorphism. Different searches and analysis strategies might contribute to this finding.

We found that not all control data in these studies were population-based. After removing studies with hospitalbased control data, the same positive correlation between the CYP4AF2 rs1558139 C/T polymorphism and risk of cardiovascular and cerebrovascular diseases was obtained under allele, heterozygote and dominant genetic models. In addition, sensitivity analysis indicated the stability of the statistical results. Nevertheless, the small sample size of the included case-control studies, to a certain extent, limits the statistical power. Limited data were extracted for the CYP4AF2 rs1558139 C/T polymorphism subgroup with the diseases of ischemic stroke or myocardial infarction.

Although no high degree of heterogeneity was observed in all meta-analyses of the CYP4AF2 rs1558139 polymorphism, high heterogeneity was observed in most of the overall meta-analyses of CYP4AF2 rs2108622. Apart from coronary heart disease and hypertension subgroup analyses, high heterogeneity still existed in other "Asian", "ischemic stroke" and "population-based" subgroups. The complexity of disease feature might be the source of the large publication bias. In addition, unpublished data or 


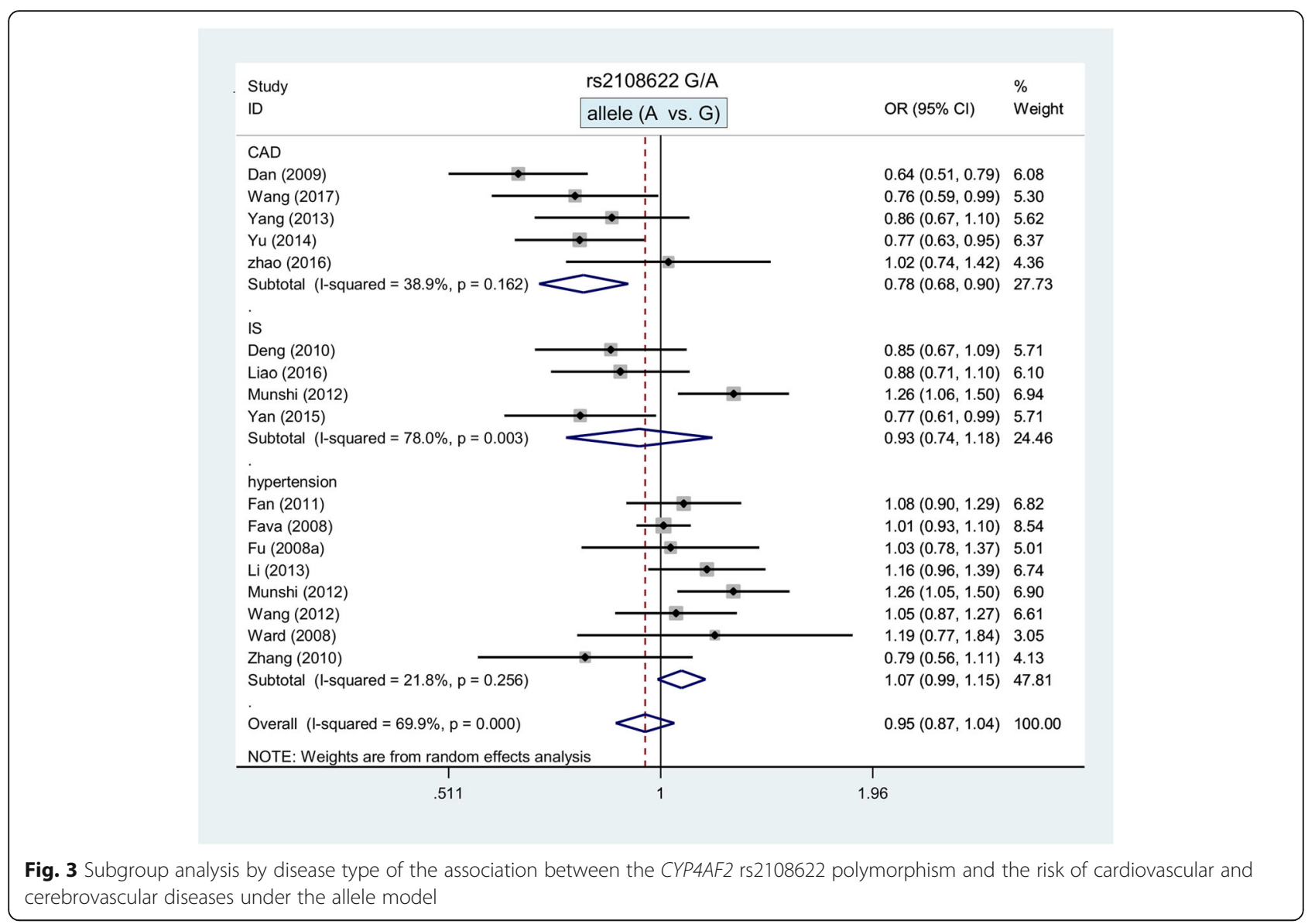

articles in other languages may bias the selection of the studies analyzed.

Given the complicated etiologies in different types of cardiovascular and cerebrovascular diseases [4, 39], additional relative factors, such as obesity, age, comorbidities, smoking, and drinking should be assessed. We spared no effort to retrieve the relative articles using the general terms and the specific forms of heart, cardiovascular and cerebrovascular diseases (e.g., myocardial infarction, coronary artery disease, or hypertension, etc.). However, limited data of the above relative factors were obtained. Only four case-control studies $[8,19,25,27]$ were enrolled

Table $\mathbf{3}$ The assessment of publication bias

\begin{tabular}{|c|c|c|c|c|c|}
\hline \multirow[t]{2}{*}{ SNP } & \multirow[t]{2}{*}{ Comparison } & \multicolumn{2}{|c|}{ Begg's test } & \multicolumn{2}{|c|}{ Egger's test } \\
\hline & & $z$ & $P$ value & $t$ & $P$ value \\
\hline \multirow[t]{5}{*}{ rs1558139 C/T } & allele (T vs. C) & 0.89 & 0.371 & -0.19 & 0.853 \\
\hline & homozygote (TT vs. CC) & 1.07 & 0.283 & -0.74 & 0.482 \\
\hline & heterozygote (CT vs. CC) & 0.72 & 0.474 & 0.66 & 0.526 \\
\hline & dominant (CT + TT vs. CC) & 0.89 & 0.371 & 0.63 & 0.549 \\
\hline & recessive (TT vs. CC $+C T$ ) & 1.07 & 0.283 & -1.33 & 0.221 \\
\hline \multirow[t]{5}{*}{ rs $2108622 \mathrm{G} / \mathrm{A}$} & allele (A vs. G) & 1.69 & 0.091 & -1.18 & 0.255 \\
\hline & homozygote (AA vs. GG) & 1.28 & 0.202 & -1.26 & 0.227 \\
\hline & heterozygote (GA vs. GG) & 1.85 & 0.064 & -1.55 & 0.143 \\
\hline & dominant (GA + AA vs. GG) & 1.11 & 0.266 & -1.18 & 0.257 \\
\hline & recessive (AA vs. GG + GA) & 0.78 & 0.434 & 0.57 & 0.579 \\
\hline
\end{tabular}



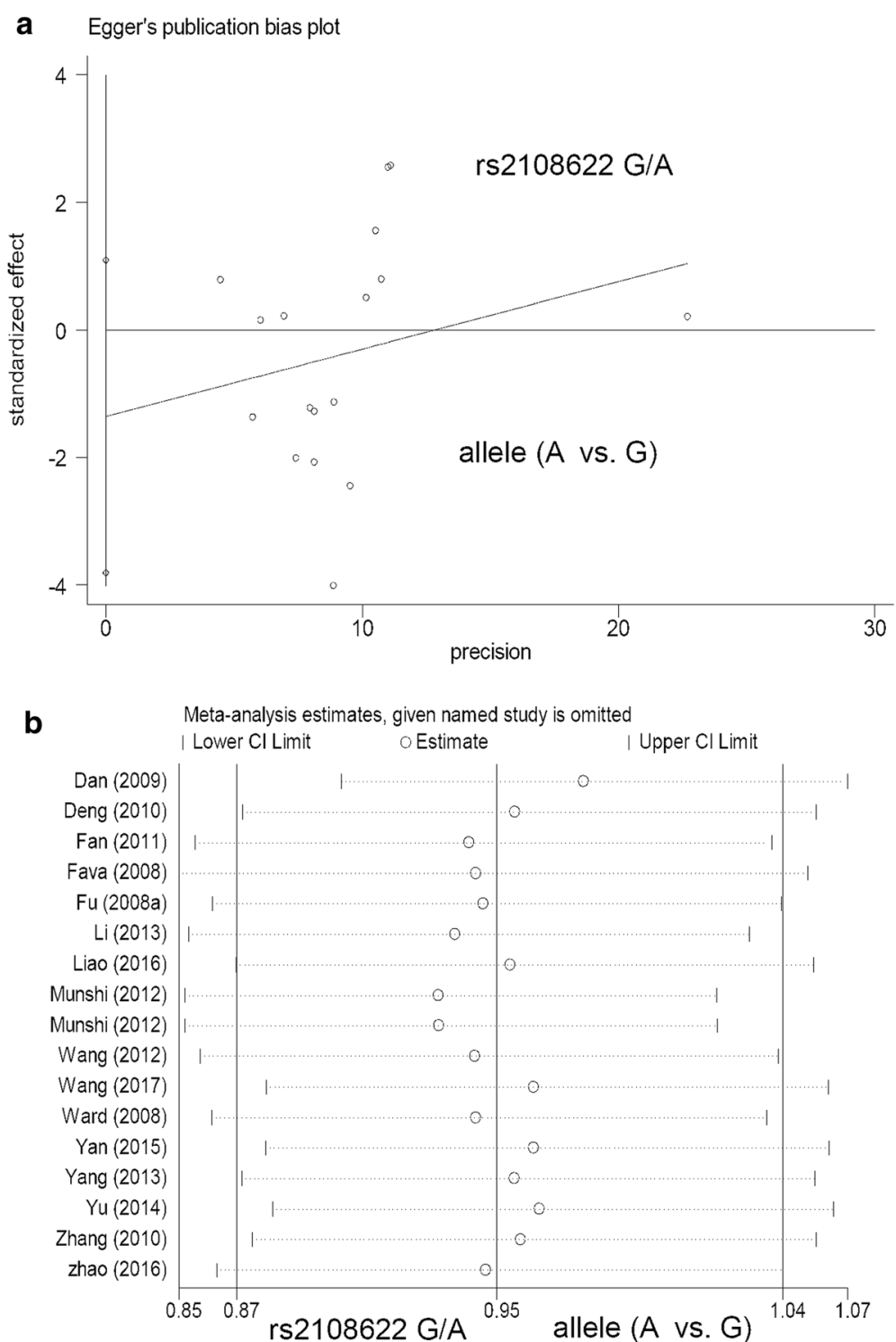

Fig. 4 Begg's test and sensitivity analysis for the CYP4AF2 rs2108622 polymorphism under the allele model. a Funnel plot of Egger's test; $\mathbf{b}$ Sensitivity analysis

to perform the male/female subgroup analysis for the association between CYP4AF2 rs1558139 and hypertension risk, while six studies $[8,19,20,23,27,32]$ were enrolled for CYP4F2 rs2108622.

Adequate data on the genotype frequencies of both case and control studies are necessary to conduct SNP meta-analyses or subgroup analyses. Thus, only two SNPs (rs1558139, rs2108622) were studied in the present metaanalysis based on the available data. We did not examine the role of other variants within the CYP4F2 gene, such as rs3093100, rs3093105, rs3093166, and rs3093135. The "G/G/G/T" haplotype of rs2108622-rs3093100-rs3093105rs3093135 has been associated with an increased risk of coronary artery disease, however the "G/G/T/A" haplotype was associated with a reduced risk of coronary heart disease [9]. The potential distinct effect of different haplotypes merits further study. In addition, the combined effect of CYP4F2 and other genes, such as CYP4A11 (Cytochrome P450 Family 4 Subfamily A Member 11), should be analyzed upon the publication of sufficient data.

\section{Conclusions}

Overall, the C/T genotype of the CYP4AF2 rs1558139 polymorphism might serve as a protective factor for male patients with hypertension in Asian populations, and CYP4AF2 rs2108622 may confer reduced genetic susceptibility to coronary heart disease. 


\section{Additional files}

Additional file 1: Table S1-S7. Table S1. Details of the search strategy based on four databases. Table S2. Basic information of the eligible studies in the meta-analysis. Table S3. Data of NOS assessment system. Table S4. Genotype frequencies of the eligible studies in the metaanalysis. Table S5. Genotype frequencies of the male/female group in some eligible studies. Table S6. Subgroup analysis by gender for the association between CYP4AF2 rs1558139 and hypertension risk. Table S7. Subgroup analysis by gender for the association between CYP4AF2 rs2108622 and hypertension risk. (DOCX 63 kb)

Additional file 2: Figure S1. Subgroup analysis by disease type of the association between the CYP4AF2 rs1558139 polymorphism and the risk of cardiovascular and cerebrovascular diseases under the allele ( $T$ vs. C) model.. (TIFF 887 kb)

Additional file 3: Figure S2. Subgroup analysis by disease type of the association between the CYP4AF2 rs1558139 polymorphism and the risk of cardiovascular and cerebrovascular diseases under the dominant $(C T+$ TT vs. CC) model. (TIFF 967 kb)

Additional file 4: Figure S3. Subgroup analysis by gender of the association between the CYP4AF2 rs1558139 polymorphism and the risk of cardiovascular and cerebrovascular diseases under the allele (T vs. C) model. (TIFF $1958 \mathrm{~kb}$ )

Additional file 5: Figure S4. Subgroup analysis by disease type of the association between the CYP4AF2 rs2108622 polymorphism and the risk of cardiovascular and cerebrovascular diseases under the homozygote (AA vs. GG) model. (TIFF 1760 kb)

Additional file 6: Figure S5. Subgroup analysis by disease type of the association between the CYP4AF2 rs2108622 polymorphism and the risk of cardiovascular and cerebrovascular diseases under the heterozygote (GA vs. GG) model. (TIFF 1717 kb)

Additional file 7: Figure S6. Subgroup analysis by disease type of the association between the CYP4AF2 rs2108622 polymorphism and the risk of cardiovascular and cerebrovascular diseases under the dominant (GA + AA vs. GG) model. (TIFF 1689 kb)

Additional file 8: Figure S7. Subgroup analysis by disease type of the association between the CYP4AF2 rs2108622 polymorphism and the risk of cardiovascular and cerebrovascular diseases under the recessive (AA vs. $\mathrm{GG}+\mathrm{GA}$ ) model. (TIFF $1792 \mathrm{~kb}$ )

Additional file 9: Figure S8. Subgroup analysis by gender of the association between the CYP4AF2 rs2108622 polymorphism and the risk of cardiovascular and cerebrovascular diseases under the allele (A vs. G) model. (TIFF $1045 \mathrm{~kb}$ )

\section{Abbreviations}

20-HETE: 20-hydroxy eicosane arachidonic acid; AA: Arachidonic acid; CAD: Coronary artery disease; Cls: Confidence intervals; CYP4A11: Cytochrome P450 Family 4 Subfamily A Member 11: CYP4F2: Cytochrome P450 Family 4 Subfamily F Member 2; IS: Ischemic stroke; MI: Myocardial infarction; OR: Odds ratio; PRISMA: Preferred Reporting Items for Systematic Reviews and MetaAnalyses; SNPs: Single nucleotide polymorphisms

\section{Acknowledgments}

Not applicable.

\section{Funding}

Not applicable.

\section{Availability of data and materials}

All data generated or analyzed during the present study are included in this published article

\section{Authors' contributions}

TZ designed the study. TZ, KY and XL extracted, analyzed, and interpreted the data. TZ and KY drafted the manuscript. All authors read and approved the final version of the manuscript.
Ethics approval and consent to participate

Not applicable.

\section{Consent for publication}

Not applicable.

\section{Competing interests}

The authors declare that they have no competing interests.

\section{Publisher's Note}

Springer Nature remains neutral with regard to jurisdictional claims in published maps and institutional affiliations.

\section{Author details}

${ }^{1}$ First Department of Neurology, The First Hospital of Zibo, No.4 Emei Mountain Road, Boshan District, Zibo City, Shandong 255200, People's Republic of China. ${ }^{2}$ China Medical University Hospital of Boshan District, Zibo City, Shandong 255200, People's Republic of China.

Received: 6 December 2017 Accepted: 29 January 2018

Published online: 09 February 2018

\section{References}

1. Koberle B, Koch B, Fischer BM, Hartwig A. Single nucleotide polymorphisms in DNA repair genes and putative cancer risk. Arch Toxicol. 2016;90(10): 2369-88.

2. Srinivasan S, Clements JA, Batra J. Single nucleotide polymorphisms in clinics: fantasy or reality for cancer? Crit Rev Clin Lab Sci. 2016;53(1):29-39.

3. Chauhan G, Debette S. Genetic Risk factors for ischemic and hemorrhagic stroke. Curr Cardiol Rep. 2016;18(12):124.

4. Della-Morte D, Pacifici F, Rundek T. Genetic susceptibility to cerebrovascular disease. Curr Opin Lipidol. 2016:27(2):187-95.

5. van der Harst $P$, de Windt $L$, Chambers JC. Translational perspective on epigenetics in cardiovascular disease. J Am Coll Cardiol. 2017;70(5):590-606.

6. Ehret GB. Genome-wide association studies: contribution of genomics to understanding blood pressure and essential hypertension. Curr Hypertens Rep. 2010;12(1):17-25

7. Levy D, Ehret GB, Rice K, Verwoert GC, Launer LJ, Dehghan A, et al. Genome-wide association study of blood pressure and hypertension. Nat Genet. 2009;41(6):677-87.

8. Fu Z, Nakayama T, Sato N, Izumi Y, Kasamaki Y, Shindo A, et al. Haplotypebased case-control study of the human CYP4F2 gene and essential hypertension in Japanese subjects. Hypertens Res. 2008a;31(9):1719-26.

9. Yu C, Yan Q, Fu C, Shi W, Wang H, Zeng C, et al. CYP4F2 genetic polymorphisms are associated with coronary heart disease in a Chinese population. Lipids Health Dis. 2014;13:83.

10. Waldman M, Peterson SJ, Arad M, Hochhauser E. The role of 20-HETE in cardiovascular diseases and its risk factors. Prostaglandins Other Lipid Mediat. 2016:125:108-17.

11. Wu CC, Gupta T, Garcia V, Ding Y, Schwartzman ML. 20-HETE and blood pressure regulation: clinical implications. Cardiol Rev. 2014;22(1):1-12

12. Hoopes SL, Garcia V, Edin ML, Schwartzman ML, Zeldin DC. Vascular actions of 20-HETE. Prostaglandins Other Lipid Mediat. 2015;120:9-16.

13. Luo XH, Li GR, Li HY. Association of the CYP4F2 rs2108622 genetic polymorphism with hypertension: a meta-analysis. Genet Mol Res. 2015; 14(4):15133-9.

14. Meng C, Wang J, Ge WN, Tang SC, Xu GM. Correlation between CYP4F2 gene rs2108622 polymorphism and susceptibility to ischemic stroke. Int J Clin Exp Med. 2015;8(9):16122-6.

15. Moher D, Liberati A, Tetzlaff J, Altman DG. Preferred reporting items for systematic reviews and meta-analyses: the PRISMA statement. PLoS Med. 2009;6(7):e1000097.

16. Stang A. Critical evaluation of the Newcastle-Ottawa scale for the assessment of the quality of nonrandomized studies in meta-analyses. Eur J Epidemiol. 2010:25(9):603-5.

17. Dan SL, Lin RY, Zou XX. Association of CYP4F2 gene polymorphism and haplotypes with coronary heart disease in Chongqing Han. Chin J Geriatr Heart Brain Ves Dis. 2009;11(7):505-8.

18. Deng SM. Effect of polymorphisms of CYP4F2, CYP4A11 and LTC4S gene on ischemic stroke. China Medical University. 2010;38-43. 
19. Fan $\mathrm{CH}$, He QF, Yu M, Chen ZK, Zhong YH. Association between genetic polymorphisms of CYP4F2 and susceptibility of essential hypertension. Zhejiang Med J. 2011;33(12):1732-4.

20. Fava C, Montagnana M, Almgren P, Rosberg L, Lippi G, Hedblad B, et al. The V433M variant of the CYP4F2 is associated with ischemic stroke in male swedes beyond its effect on blood pressure. Hypertension. 2008;52(2):37380.

21. Fu Z, Nakayama T, Sato N, Izumi Y, Kasamaki Y, Shindo A, et al. A haplotype of the CYP4F2 gene is associated with cerebral infarction in Japanese men. Am J Hypertens. 2008b;21(11):1216-23.

22. Huang D, Fu ZY, Yang YN, Xie X, Wang YH, Ma YT. Association on the haplotypes of CYP4F2 gene and myocardial infarction. Zhonghua Liu Xing Bing Xue Za Zhi. 2009;30(7):733-6.

23. Li M, Wang H, Liu JL, Wang $\sqcup$, Zhang B, Liu Y, et al. Association of rs2108622 polymorphism of CYP4F2 gene and essential hypertension in the northern Han Chinese. Journal of cardiovascular and pulmonary. Diseases. 2013;32(5):538-42.

24. Liao D, Yi X, Zhang B, Zhou Q, Lin J. Interaction between CYP4F2 rs2108622 and CPY4A11 rs9333025 variants is significantly correlated with susceptibility to ischemic stroke and 20-Hydroxyeicosatetraenoic acid level. Genet Test Mol Biomarkers. 2016;20(5):223-8.

25. Liu JH. Association of CYP4A11 and CYP4F2 polymorphism with essential hypertension and coronary heart disease. Southern Medical University. 2012; 62-8.

26. Munshi A, Sharma V, Kaul S, Al-Hazzani A, Alshatwi AA, Shafi G, et al. Association of $1347 \mathrm{G} / \mathrm{a}$ cytochrome P450 4F2 (CYP4F2) gene variant with hypertension and stroke. Mol Biol Rep. 2012;39(2):1677-82.

27. Wang F. Effect of common polymorphisms in CYP4A11, CYP4F2 genes on the presence of essential hypertension. Ningbo University. 2012;9-20.

28. Wang XH, Zhang WK, Fu W, Cao HK. Study of the correlation between the gene polymorphism of CYP4F2 and coronary heart disease in Yunnan Han population. Journal of Bengbu Medical College. 2017;42(5):565-7.

29. Ward NC, Tsai IJ, Barden A, van Bockxmeer FM, Puddey IB, Hodgson JM, et al. A single nucleotide polymorphism in the CYP4F2 but not CYP4A11 gene is associated with increased 20-HETE excretion and blood pressure. Hypertension. 2008;51(5):1393-8.

30. Yan HQ, Yuan Y, Zhang P, Huang Z, Chang L, Gui YK. CYP4F2 gene single nucleotide polymorphism is associated with ischemic stroke. Genet Mol Res. 2015;14(1):659-64.

31. Yang H. Association study of haplotypes of the CYP gene and sex hormone with coronary heart disease. Master: Xinjiang Medical University; 2013.

32. Zhang LX, Yang J, Chen FF, Wang DW. Association between the G20597A variant of CYP4F2 gene and essential hypertension. J Chin Physician. 2010; 12(1):27-30.

33. Zhao P, Chang PY, Jiang K, Ge RL. The association between CYP4F2 gene polymorphism and coronary heart disease. Chin J Gerontol. 2017;37(15): 3703-5.

34. Zhao P, Chang PY, Nan JL, Jiang P, Ge RL, Xie JM. Research for correlation between CYP4F2 gene polymorphism and coronary heart disease in Mongolian patients. Chinese Journal of cardiovascular Rehabilitation Medicine. 2016;25(1):18-21.

35. Sayols-Baixeras S, Lluis-Ganella C, Lucas G, Elosua R. Pathogenesis of coronary artery disease: focus on genetic risk factors and identification of genetic variants. Appl Clin Genet. 2014;7:15-32.

36. Stec DE, Roman RJ, Flasch A, Rieder MJ. Functional polymorphism in human CYP4F2 decreases 20-HETE production. Physiol Genomics. 2007;30(1):74-81.

37. Cassella CR, Jagoda A. Ischemic stroke: advances in diagnosis and management. Emerg Med Clin North Am. 2017;35(4):911-30.

38. Ding H, Cui G, Zhang L, Xu Y, Bao X, Tu Y, et al. Association of common variants of CYP4A11 and CYP4F2 with stroke in the Han Chinese population. Pharmacogenet Genomics. 2010:20(3):187-94.

39. Jokinen E. Obesity and cardiovascular disease. Minerva Pediatr. 2015;67(1):25-32.

\section{Submit your next manuscript to BioMed Central and we will help you at every step:}

- We accept pre-submission inquiries

- Our selector tool helps you to find the most relevant journal

- We provide round the clock customer support

- Convenient online submission

- Thorough peer review

- Inclusion in PubMed and all major indexing services

- Maximum visibility for your research

Submit your manuscript at www.biomedcentral.com/submit
Biomed Central 